\title{
Geochemical characteristics and evolution of REE in the Early Precambrian sediments : evidence from the southern margin of the North China Craton
}

\author{
*Department of Geologi, Peking University, Beijing 100871
}

The REE characteristics of both the chemical and the clastic sediments from six early Precambrian terrains at the southern margin of the North China craton show that the sedimentary Eu-depletion started from about 2300 $M a$ and the values of $\Sigma R E E$ of the sediments less than $2300 \mathrm{Ma}$ are higher than those of the sediments more than $2300 \mathrm{Ma}$. These phenomena can be logically ascribed to considering the changes in surficial environment and interpreted by using the new HSAB (hard and soft acids and bases) theory. We argue that the sediments deposited in anoxic environment before $2300 \mathrm{Ma}$ are characterized by positive Eu-anomaly, low $\sum R E E$ and occasionally high $L a_{N} / Y b_{N}$; whereas the sediments in oxidic condition after 2300 Ma show Eu-depletion, high $\sum R E E$ and low $L a_{N} / Y b_{N}$. Possibly, the surficial environment (including atmosphere) turned to be oxidic at about $2300 \mathrm{Ma}$. Hence, the REE can be used to indicate the nature of the environment.

\section{Introduction}

At the beginning of the Proterozoic, significant changes took place in many respects of the earth (Condie, 1981, 1982. 1993; Kroner, 1981; Frakes, 1979; Schopf, 1977; Eriksson and Truswell, 1978; Hu, 1988; Sun, 1984; Chen, 1989. 1990; Chen et al., 1991a, 1994, 1996; Taylor and Mclennan. 1985; Mclennan and Taylor, 1991). Taylor and Mclennan (1985) advanced that the Archean sediments showed clear negative Eu-anomaly. Eu-depletion of the post-Archean sediments is attributed to the extensive development of K-granite at the end of the Archean. By Taylor's model, the REE characteristics of the sediments are controlled by their provenance rather than the process of weathering-deposition-diagenesis-metamorphism or the sedimentary environments. Through the study of the southern margin of the North China craton, we find that, besides the provenance of sediments, the sedimentary environment is a chief factor affecting the characteristics of REE in the sediments. Based on the HSAB (hard and soft acids and bases) theory (Dai, 1987) and the consideration of the changes in sedimentary environment, this paper gives an interpretation of the geochemical evolution of REE in the sediments from the southern margin of the North China craton and establishes a new model to explain the differences in REE between the Archean and the post-Archean sediments.

\section{Geology and stratigraphy}

The southern margin of the North China craton comprises the Huaxiong block and the Songii block (Figure 1). The Huaxiong block is made up of the Xiaoshan terrain and the Taihua united terrain which includes the Lushan, Wuyang, Xiong'ershan and Xiaoqinling Early Precambrian terrains. The Songshan and Jishan Early Precambrian terrains are exposed in the Songji block (Figure 1). The stratigraphy and tectonic evolution of the some terrains are generalized (Table 1 , Figure 2) on the basis of a comprehensive work including field investigation and study of petrology, geochemistry, geochronology, stratigraphy, etc. (Chen, 1987; Chen et al., 1988, 1989, 1991b; Chen and $\mathrm{Fu}, 1992$ ). These terrains were developed during the period from $3000 \mathrm{Ma}$ to $2100 \mathrm{Ma}$. The Archean-Proterozoic transition in the area in question occurred $2300 \mathrm{Ma}$ ago.

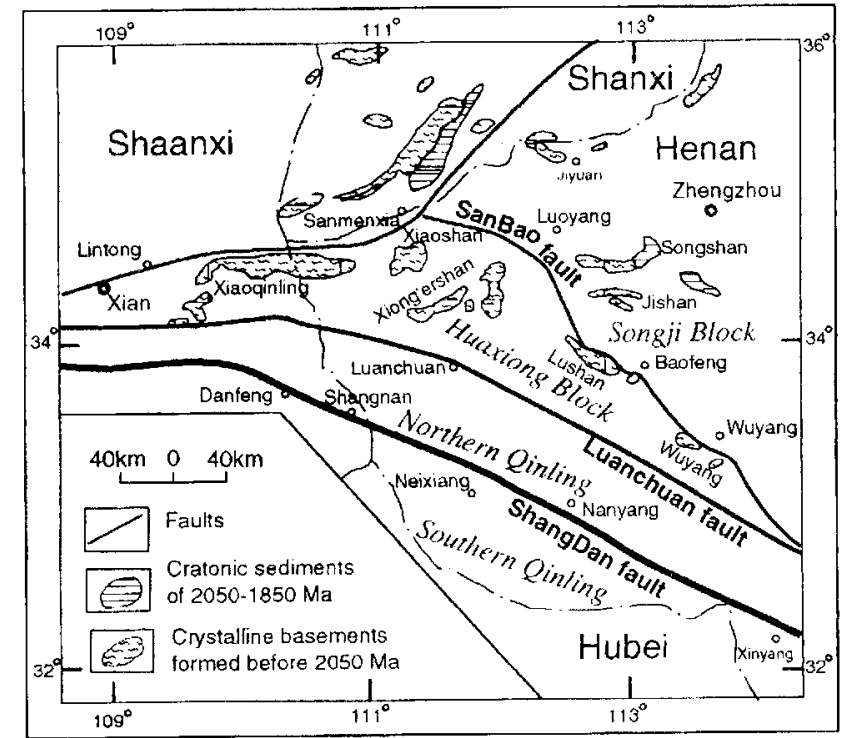

Figure 1 Distribution of early Precambrian terrains at the southern margin of the North China craton

All the rocks formed before 2300 Ma are similar to the typical Archean rock associations described by Condie (1982) such as the greenstone belts and the TTG (tonalite-trondhjemite-granodiorite) suites. As discontinuous inclusions (relicts) in the Shipaihe complex 
or the Yuyalo complex, the Qinyanggou type greenstone belts were developed before $3000 \mathrm{Ma}$ in the Songji block. The Beizi type greenstone belts in the Huaxiong block were developed during the period from $3000 \mathrm{Ma}$ to $2550 \mathrm{Ma}$. Both the Dangzehe type greenstone belts in the Huaxiong block and the Junzhao type greenstone belts in the Songii Block were formed within the range of 2550-2300 Ma (Chen et al., 1988). The Shipaihe complex in Songshan terrain and the Yuyao complex in the Jishan terrain are TTG complexes, and most of them intruded during the period from $3000 \mathrm{Ma}$ to $2550 \mathrm{Ma}$.

The supracrustal successions formed after $2300 \mathrm{Ma}$ are much similar to the typical Proterozoic supracrustal successions defined by Condie (1982). The khondalite series of the Shuidigou Group in the Huaxiong block, formed from $2300 \mathrm{Ma}$ to $2200 \mathrm{Ma}$, are the high grade metamorphic equivalent of the quartzite-carbonate-shale assemblage which was regarded as Proterozoic supracrustal assemblage 1 (Condie, 1982 ). The Angou Group in the Jishan terrain and the Xiaoshan Group in the Xiaoshan terrain are made up of the typical greenschist facies bimodal volcanics-quartzite-arkose (Proterozoic supracrustal assemblage 2): they developed during the period of 2300-2050 Ma. The Angou Group in the Songshan terrain is mainly made up of flyschoid sediments, with minor bimodal volcanics. Obviously, the intermontane molasses of the Tietonggou Group in Xiaoshan terrain and the intracratonic clastic sediments of the Songshan Group in the Songji block, formed in the period of $2050-1850$ $\mathrm{Ma}$ are sedimentary strata composed of highly matured clastics. Therefore, at the southern margin of the North China craton, the vol-

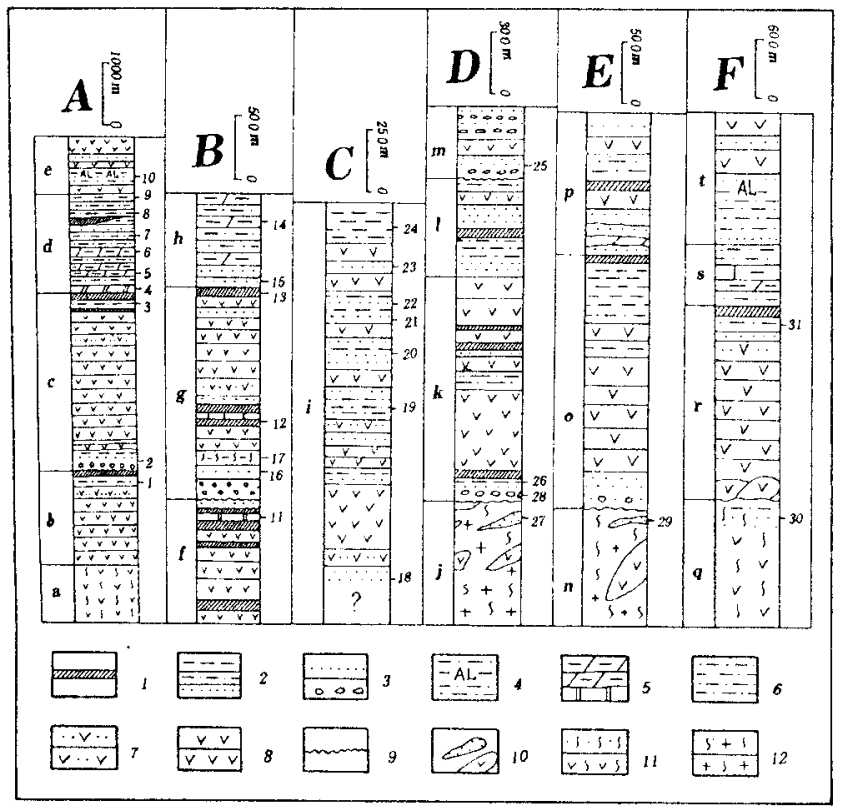

Figure 2 Schematic stratigraphic sequences and sample locations for the six terrains. Sample numbers on the right sides of each column are consistent with the numbers in Table 2 and Figures 3 7.

A: Lushan; B: Wuyang:

D: Songshan; E: Jishan;

1: banded iron formation;

3: coarse clastic sediment:

C: Xiaoshan:

F: Xiong'ershan.

2: fine clastic sediment;

4: Al-rich sediment;

5: carbonates including pelitic limestone, carboniferous limestone, chert-bearing limestone and dolomite:

6: carboniferous shale:

8: volcanics;

7: volcaniclastic sediment;

9: unconformity:

10: supracrustal rock relicts (sediments and volcanics);

11: migmatites from supracrustal rocks; 12: migmatized plutons.

a, b, f, q: Beizi Gp.;

d, e, h, s, t: Shuidigou Gp.;

j, n: Shipaihe Complex;

c, g, r: Dangzehe Gp.:

$\mathrm{m}$, p: Angou Gp. canism gap began from $2300 \mathrm{Ma}$ and ended before $1850 \mathrm{Ma}$, which is consistent with that in the other continents (Kroner, 1981).

The reasons why we propose $2300 \mathrm{Ma}$ as the Archean-Proterozoic boundary are: (1) More than 25 isotopic ages for the Archean lithologic associations are older than $2300 \mathrm{Ma}$, whereas all the ages for the Proterozoic rocks (more than 55) are less than $2300 \mathrm{Ma} \mathrm{(Hu}$. 1988, Chen, 1987, Kroner et al., 1988, Li et al., 1986). (2) The four oldest $\mathrm{K}$-Ar ages for the pegmatite veins intruded into the Archean rocks in the Songshan terrain range from $2122 \mathrm{Ma}$ to $2299 \mathrm{Ma}(\mathrm{Hu}$, 1988). (3) The isotopic ages for the rocks at the southern margin of the North China craton are concentrated at 2250-2300 Ma in the isotopic age histogram. (4) Information about geology, petrology, tectonics, environment, etc. indicates a significant geologic event occurring at about $2300 \mathrm{Ma}$.

\section{Samples and analysis}

Table 2 shows the chemical compositions of 31 samples of the sediments from the six terrains. These samples generally represent the major types of sediments in each stage before $2050 \mathrm{Ma}$ (Tab. 2. Figure 2). REE data were obtained by means of ICP analysis in Chemical Analysis and Research School of Hubei Province. The analysis precision is estimated less than $4 \%$ for every rare earth element. The analysis accuracy is evaluated within 1 ppm for total REE.

\section{Characterisites and evolution of REE in the sediments}

\section{Lushan terrain}

Sample no. 1 and no. 2 are clastic sediments older than $2300 \mathrm{Ma}$ (Figure 2A); and they show positive Eu-anomalies (Figure 3). Sample no.3, the clastic sediment, shows a slight Eu-depletion; and sample no.4, the chemical-clastic sediment, shows no Eu-anomaly (Figure 3 ). Both of them were formed at about 2300 Ma. All samples of the sediments less than $2300 \mathrm{Ma}$ in age (sample no.6: chemical sediment; no. 8 and no. 10: clastic sediments; no.5, no.7 and no.9: chemical-clastic sediments) are characterized by Eu-depletion (Figure 3)

\section{Wuyang terrain}

As sbown in Figures 2B and 4 and Tables 1 and 2, the clastic sediment (no.15) and the chemical-clastic sediment (no. 14) less than $2300 \mathrm{Ma}$ are characterized by Eu depletion (Figure 4). Being the BIF about $2300 \mathrm{Ma}$ in age, sample no. 13 is hardly depleted in Eu. All the chemical (nos.11 and 12) and the clastic (nos.16 and 17) sediments formed before $2300 \mathrm{Ma}$ show positive Eu-anomalies except sample no. 7 which was migmatized and now shows Eu-depletion (Figure 4).

\section{Xiaoshan terrain}

Seven samples (Nos. 18-24) from the Xiaoshan Group (Figure 2C) are metamorphic mudstones or volcanoclastic sediments. They are marked with clear Eu-depletion (Eu/Eu* from 0.44 to 0.78 ) and high ¿REE (from 159.844 ppm to 407.75 ppm) (Figure5).

\section{Songshan terrain}

Sample no. 25, in Figure 2D, is the sediment from the Angou Group and its Eu-depletion and high $\sum$ REE features are manifested clearly in Figure 6. Sample no.26 is the matrix of the basal conglomerate at the bottom of the Junzhao Group. Sample no.28, from the basal conglomerate, is the gravel of sedimentary rocks. Sample no. 27 is the restite of sedimentary rocks within the Shipaihe complex, which might be the source for sample no.28. Sample nos. 26, 27 and 28 
Table I Stratigraphy and tectonic evolution of some blocks and terrains

\begin{tabular}{|c|c|c|c|}
\hline \multirow[t]{2}{*}{ Geologic time } & \multicolumn{2}{|c|}{ Huaxing block } & \multirow[t]{2}{*}{ Songji block } \\
\hline & Taihua united terrain & Xiaoshan Terrain & \\
\hline $1400-1850 \mathrm{Ma}$ & cover: Xiong'er Gp. volcanics & cover: Xiong'er Gp. volcanics & uplifting, erosion \\
\hline Zhongyue movement & \multicolumn{3}{|c|}{$\begin{array}{l}\text { formation of the southern margin of the North China craton } \\
\text { amalgamation of the Huaxiong block with Songji block }\end{array}$} \\
\hline $1850-2150 \mathrm{Ma}$ & $\begin{array}{c}\text { Tietonggou Gp. intramontane } \\
\text { molasses }\end{array}$ & $\begin{array}{c}\text { Tietonggou Gp. intramontane } \\
\text { molasses }\end{array}$ & $\begin{array}{l}\text { Songshan Gp. cratonic } \\
\text { sediments }\end{array}$ \\
\hline Songyang Movement & $\begin{array}{c}\text { amalgamation with the Xiaoshan } \\
\text { terrain }\end{array}$ & $\begin{array}{l}\text { amalgamation with the Taihua } \\
\text { united terrain }\end{array}$ & close of the Angou-Xiaoshan rift \\
\hline $2 \mathrm{~J} 50-2300 \mathrm{Ma}$ & Shuidigou Gp. khondalite series & $\begin{array}{l}\text { Xiaoshan Gp. } \\
\text { bimodal volcanics }\end{array}$ & $\begin{array}{l}\text { Angou Gp. } \\
\text { bimodal volcanics }\end{array}$ \\
\hline Guojiayao Movement & $\begin{array}{l}\text { catastrophe in surficial } \\
\text { environment: } \\
\text { cratonization of Huaxiong block }\end{array}$ & $\begin{array}{l}\text { diverge from Songji; } \\
\text { metamorphism } \\
\text { and migmatization }\end{array}$ & $\begin{array}{l}\text { rifting; metamorphism and } \\
\text { pegmatite intrusion }\end{array}$ \\
\hline $2300-2550 \mathrm{Ma}$ & $\begin{array}{l}\text { Dangzehe Gp. } \\
\text { secondary greenstones }\end{array}$ & $\begin{array}{l}\text { Tianyemiao Complex } \\
\text { migmatite-granitoids }\end{array}$ & $\begin{array}{l}\text { Junzhao Gp. } \\
\text { secondary greenstones }\end{array}$ \\
\hline Shipaihe Movement & cratonization & $?$ & $\begin{array}{l}\text { cratonization and accretion of } \\
\text { Songji sialic nucleus }\end{array}$ \\
\hline $2550-3000 \mathrm{Ma}$ & $\begin{array}{c}\text { Beizi Gp. } \\
\text { greenstones developed on } \\
\text { oceanic setting }\end{array}$ & $?$ & $\begin{array}{l}\text { Shipaihe Complex: } \\
\text { migmatites, TTGs and high } \\
\text { grade metamorphic rocks }\end{array}$ \\
\hline $\begin{array}{l}\text { Qingyanggou } \\
\text { Movement }\end{array}$ & $\begin{array}{l}\text { rifting of primary simatic crust, } \\
\text { coming into being of a proto ocean }\end{array}$ & $?$ & $\begin{array}{l}\text { Intrusion of TTGs such as } \\
\text { Shipaihe and Yuyao masses }\end{array}$ \\
\hline$>3000 \mathrm{Ma}$ & $?$ & $?$ & Qingyanggou greenstones \\
\hline
\end{tabular}

Table 3 REE behavior in waters and in sediments and their relationship with the nature of environment

Nature of environment

\section{$\mathrm{fO}_{2}$}

$\mathrm{Eu}^{3+} / \mathrm{Eu}^{2+}$

species of Eu

ligands (generally anions) for coordination

sequence of REE to coordinate and precipitate cations into complexes and precipitated cations tend to retain in water sedimentary REE feature

\section{Oxidic environment}

high

high

predominance of $\mathrm{Eu}^{3+}$

hard bases such as $\mathrm{OH}^{-}, \mathrm{CO}_{3}^{2-}, \mathrm{SO}_{4}^{2-}$, and $\mathrm{NO}^{3}$

$\mathrm{HR}^{3+}>\mathrm{LR}^{3+}>\mathrm{Eu}^{2+}$

hard acids such as $\mathrm{Eu}^{3+}, \mathrm{R}^{3+}$

soft acids such as $\mathrm{Eu}^{2+}$

high $\sum R E E$, low Eu/Eu* and

low $\mathrm{LaN}_{\mathrm{N}} / \mathrm{Yb}_{\mathrm{N}}$

\section{Reducing environment}

low

low

predominance of $\mathrm{Eu}^{2+}$

soft bases such as $\mathrm{HS}^{-}, \mathrm{S}^{--}, \mathrm{SCN}^{-}$, $\mathrm{S}_{2} \mathrm{O}_{3}{ }^{2-}$ and $\mathrm{CO}$

$\mathrm{Eu}^{2+}>\mathrm{LR}^{3+}>\mathrm{HR}^{3+}$

soft acids such as $\mathrm{Eu}^{2+}$

hard acids such as $\mathrm{Eu}^{3+}, \mathrm{R}^{3+}$

low $\sum R E E$, high Eu/Eu* and

high $\mathrm{LaN}_{\mathrm{N}} / \mathrm{Yb}_{\mathrm{N}}$ 


\begin{tabular}{|c|c|c|c|c|c|c|c|c|c|c|c|c|c|c|c|c|c|c|c|c|c|c|c|c|c|c|c|c|c|}
\hline & $\mathrm{SiO}_{2}$ & $\mathrm{TiO}_{2}$ & $\mathrm{Al}_{2} \mathrm{O}_{3}$ & $\mathrm{Fe}_{2} \mathrm{O}_{3}$ & $\mathrm{FeO}$ & $\mathrm{MnO}$ & Mgo & $\mathrm{CaO}$ & $\mathrm{Na}_{2} \mathrm{O}$ & $\mathrm{K}_{2} \mathrm{O}$ & $\mathrm{P}_{2} \mathrm{O}_{5}$ & Other & $\mathrm{La}$ & $\mathrm{Ce}$ & $\operatorname{Pr}$ & Nd & $\mathrm{Sm}$ & Eu & Gd & Tb & Dy & Ho & $\mathrm{Er}$ & $\mathrm{Tm}$ & $\mathbf{Y b}$ & Lu & $\mathrm{La}_{\mathbf{N}} / \mathbf{Y b}_{\mathbf{N}}$ & $\mathbf{E u} / \mathbf{E} u^{*}$ & ¿REF \\
\hline 1 & 68.46 & 0.44 & 15.72 & 2.85 & 0.88 & 0.04 & 1.03 & 3.67 & 4.24 & 0.97 & 0.08 & 1.42 & 35.91 & $60 .+0$ & 7.30 & 21.21 & 3.00 & 1.22 & 1.69 & 0.30 & 0.90 & 0.19 & 0.46 & 0.08 & 0.38 & 0.05 & 54.75 & 1.52 & 133.09 \\
\hline 2 & 74.36 & 0.04 & 13.53 & 0.18 & 0.49 & 0.01 & 0.30 & 2.52 & 3.93 & 3.30 & 0.02 & 1.33 & 4.70 & 5.10 & 0.87 & 2.11 & 0.38 & 0.47 & 0.29 & 0.07 & 0.25 & (9.).94 & 0.11 & 0.02 & 0.09 & 0.01 & 30.26 & 4.20 & $\mid 4.5]$ \\
\hline 3 & 70.28 & 0.70 & 11.49 & 2.99 & 3.70 & 0.05 & 1.12 & 0.82 & 2.09 & 5.43 & 0.15 & 0.54 & 9.14 & 24.19 & 3.88 & 16.47 & 4.04 & 1.10 & 4.09 & 0.74 & 4.47 & 0.89 & 2.51 & 0.39 & 2.29 & 0.32 & 2.31 & 0.83 & 74.52 \\
\hline 4 & 57.04 & 0.53 & 14.07 & 2.19 & 3.70 & 0.05 & 5.48 & 3.73 & 1.37 & 1.67 & 0.10 & 9.89 & 14.71 & 35.22 & 4.03 & 12.18 & 2.54 & 0.77 & 2.18 & 0.41 & 2.15 & 0.43 & 1.24 & 0.19 & 1.15 & 0.16 & 7.41 & 0.99 & 77.36 \\
\hline 5 & 56.80 & 0.35 & 7.17 & 1.29 & 3.25 & 0.10 & 10.12 & 12.75 & 0.86 & 2.04 & 0.10 & 4.66 & 18.55 & 37.16 & 5.54 & 20.74 & 4.68 & 0.82 & 4.12 & 0.75 & 4.25 & 0.81 & 2.38 & 0.36 & 2.28 & 0.30 & 4.71 & 0.56 & 102.74 \\
\hline 6 & 8.66 & 0.4 .5 & 2.28 & 0.49 & 0.98 & 0.03 & 2.29 & 46.11 & 0.46 & 0.40 & 0.16 & 37.95 & 16.10 & 32.80 & 4.01 & 12.79 & 2.51 & 0.54 & 1.97 & 0.34 & 1.53 & 0.30 & 0.73 & 0.12 & 0.54 & 0.07 & .27 & 0.72 & 74.35 \\
\hline 7 & 52.60 & 0.45 & 22.08 & 1.39 & 1.85 & 0.10 & 5.03 & 3.38 & 4.24 & 3.39 & 0.10 & 5.00 & 19.32 & 42.41 & 6.01 & 22.78 & 5.07 & 0.85 & 4.57 & 0.78 & 4.33 & 0.87 & 2.54 & 0.39 & 2.49 & 0.36 & 50 & 0.53 & 112.77 \\
\hline 8 & 66.10 & 0.25 & 19.01 & 0.67 & 0.55 & 0.03 & 0.71 & 0.56 & 10.96 & 0.20 & 0.03 & 0.58 & 7.26 & 48.74 & 3.94 & 11.11 & 3.84 & 0.41 & 4.50 & 1.00 & 6.88 & 1.35 & 4.06 & 0.62 & 4.07 & 0.49 & 4.50 & 0.53 & 112.77 \\
\hline 9 & 52.32 & 0.55 & 14.37 & 6.37 & 0.43 & 0.08 & 9.30 & 6.31 & 4.68 & 0.59 & 0.10 & 3.96 & 40.29 & 68.20 & 8.53 & 26.95 & 5.08 & 0.96 & 3.81 & 0.64 & 3.10 & 0.62 & 1.71 & 0.29 & 1.65 & 0.24 & 1.03 & 0.30 & 98.27 \\
\hline 10 & 70.10 & 0.65 & 11.70 & 3.59 & 1.90 & 0.04 & 1.21 & 0.54 & 1.38 & 5.91 & 0.05 & 1.86 & 24.22 & 26.64 & 6.05 & 24.29 & 6.17 & 1.23 & 6.92 & 1.24 & 7.74 & 1.54 & 4.43 & 0.69 & 4.35 & 0.62 & 14.15 & 0.65 & 162.07 \\
\hline 11 & 3.03 & 0.02 & 0.61 & 1.12 & 1.94 & 0.00 & 19.76 & 30.37 & 0.00 & 0.00 & 0.01 & 42.24 & 2.89 & 4.16 & 0.52 & 1.50 & 0.25 & 0.10 & 0.23 & 0.04 & 0.23 & 0.06 & 0.17 & 0.03 & 0.15 & 0.03 & 3.23 & 0.58 & 116.13 \\
\hline 12 & 40.53 & 0.07 & 0.86 & 17.82 & 16.63 & 0.39 & 6.01 & 13.50 & 0.65 & 0.17 & 0.00 & 3.05 & 1.64 & 2.52 & 0.27 & 0.86 & 0.15 & 0.07 & 0.17 & 0.03 & 0.18 & 0.04 & 0.13 & 0.02 & 0.12 & 0.02 & 11.16 & 1.21 & 10.35 \\
\hline 13 & 43.63 & 0.05 & 1.07 & 27.47 & 13.73 & 0.43 & 3.41 & 6.91 & 1.00 & 0.39 & 0.00 & 1.78 & 1.98 & 3.75 & 0.47 & 1.58 & 0.28 & 0.08 & 0.24 & 0.04 & 0.22 & 0.05 & 0.12 & 0.02 & 0.13 & 0.02 & 7.85 & 1.39 & 6.22 \\
\hline 14 & 57.44 & 0.64 & 11.44 & 2.48 & 2.22 & 0.15 & 6.23 & 11.24 & 0.91 & 1.61 & 0.11 & 5.30 & 40.53 & 73.46 & 0.39 & 32.77 & 6.91 & 1.44 & 6.78 & 1.11 & 6.61 & 1.37 & 3.86 & 0.59 & 3.62 & 0.55 & 8.69 & 0.90 & 8.98 \\
\hline 15 & 62.84 & 0.63 & 13.72 & 2.65 & 3.47 & 0.11 & 2.32 & 3.00 & 2.02 & 4.08 & 0.24 & 3.07 & 62.49 & 94.85 & 10.33 & 29.92 & 4.36 & 0.88 & 3.28 & 0.49 & 2.11 & 0.41 & 1.02 & 0.15 & 0.77 & 0.11 & 6.49 & 0.64 & 188.98 \\
\hline 16 & 92.30 & 0.15 & 3.57 & 0.11 & 1.31 & 0.16 & 0.13 & 0.37 & 0.22 & 1.74 & 0.01 & 0.37 & 6.30 & 9.96 & 1.31 & 3.78 & 0.58 & 0.19 & 0.40 & 0.09 & 0.31 & 0.07 & 0.18 & 0.04 & 0.20 & 0.03 & 6.90 & 0.69 & 211.17 \\
\hline 17 & 95.15 & 0.04 & 1.28 & 0.11 & 1.43 & 0.19 & 0.00 & 0.22 & 0.22 & 0.78 & 0.01 & 0.20 & 15.72 & 26.05 & 3.20 & 10.29 & 1.73 & 0.34 & 1.48 & 0.25 & 1.66 & 0.35 & 1.06 & 0.16 & 0.98 & 0.15 & 3.53 & $\begin{array}{l}0.07 \\
1.16\end{array}$ & 23.43 \\
\hline 18 & & & & & & & & & & & & & 62.51 & 134.88 & 16.64 & 50.62 & 8.45 & 1.47 & 5.23 & 0.96 & 3.85 & 0.75 & 1.98 & 0.35 & 1.81 & 0.27 & $\begin{array}{l}18.5 .5 \\
9.26\end{array}$ & 0.63 & 63.43 \\
\hline $\begin{array}{l}19 \\
20\end{array}$ & $\begin{array}{l}69.00 \\
74.47\end{array}$ & $\begin{array}{l}0.54 \\
0.08\end{array}$ & $\begin{array}{l}14.71 \\
14.18\end{array}$ & 4.22 & $\begin{array}{l}0.46 \\
0.77\end{array}$ & $\begin{array}{l}0.04 \\
0.03\end{array}$ & $\begin{array}{l}0.63 \\
0.34\end{array}$ & $\begin{array}{l}1.38 \\
0024\end{array}$ & $\begin{array}{l}0.50 \\
2.44\end{array}$ & $\begin{array}{l}3.87 \\
4.20\end{array}$ & $\begin{array}{l}0.13 \\
0.07\end{array}$ & $\begin{array}{l}4.03 \\
1.59\end{array}$ & $\begin{array}{l}44.51 \\
42.78\end{array}$ & $\begin{array}{l}84.57 \\
70.85\end{array}$ & $\begin{array}{l}10.39 \\
9.51\end{array}$ & $\begin{array}{l}34.53 \\
28.69\end{array}$ & $\begin{array}{l}5.94 \\
4.52\end{array}$ & $\begin{array}{l}1.01 \\
0.62\end{array}$ & $\begin{array}{l}4.18 \\
2.89\end{array}$ & $\begin{array}{l}0.72 \\
0.51\end{array}$ & $\begin{array}{l}3.02 \\
2.48\end{array}$ & $\begin{array}{l}0.56 \\
0.54\end{array}$ & $\begin{array}{l}1.52 \\
1.68\end{array}$ & $\begin{array}{l}0.28 \\
0.30\end{array}$ & $\begin{array}{l}1.60 \\
1.81\end{array}$ & $\begin{array}{l}0.21 \\
0.28\end{array}$ & $\begin{array}{l}9.20 \\
20.01\end{array}$ & 0.63 & 289.77 \\
\hline $\begin{array}{l}20 \\
21\end{array}$ & 74.47 & 0.08 & & 1.06 & 0.77 & 0.03 & 0.34 & & 2.44 & 4.20 & 0.07 & 1.59 & $\begin{array}{l}42.78 \\
31.80\end{array}$ & $\begin{array}{l}70.85 \\
83.42\end{array}$ & $\begin{array}{l}9.51 \\
8.24\end{array}$ & $\begin{array}{l}28.69 \\
23.21\end{array}$ & $\begin{array}{l}4.52 \\
3.76\end{array}$ & $\begin{array}{l}0.62 \\
0.49\end{array}$ & $\begin{array}{l}2.89 \\
2.52\end{array}$ & $\begin{array}{l}0.51 \\
0.48\end{array}$ & $\begin{array}{l}2.48 \\
2.16\end{array}$ & $\begin{array}{l}0.54 \\
0.45\end{array}$ & $\begin{array}{l}1.68 \\
1.34\end{array}$ & $\begin{array}{l}0.30 \\
0.25\end{array}$ & $\begin{array}{l}1.81 \\
1.49\end{array}$ & $\begin{array}{l}0.28 \\
0.23\end{array}$ & 16.12 & 0.59 & 193.04 \\
\hline 22 & 74.42 & 0.22 & 14.17 & 1.37 & 0.96 & 0.03 & 0.93 & 0.45 & 0.45 & 4.26 & 0.02 & 2.31 & 82.55 & 231.62 & 20.04 & 51.80 & 9.05 & 1.06 & 4.99 & 0.84 & 2.11 & 0.42 & 1.21 & 0.32 & 1.41 & 0.23 & 13.69 & 0.49 & 167.46 \\
\hline 23 & 68.06 & 0.60 & 16.59 & 1.57 & 1.99 & 0.05 & 0.55 & 1.08 & 1.98 & 4.04 & 0.02 & 2.31 & 43.45 & 87.99 & 11.37 & 38.84 & 6.29 & 1.36 & 4.09 & 0.63 & 2.56 & 0.55 & 1.46 & 0.26 & 1.48 & 0.23 & 12.36 & 0.46 & 159.84 \\
\hline 24 & 71.66 & 0.25 & 14.48 & 0.70 & 2.43 & 0.10 & 0.96 & 1.00 & 6.04 & 1.60 & 0.10 & 0.44 & 52.76 & 96.32 & 11.90 & 37.47 & 5.75 & 1.12 & 3.69 & 0.60 & 2.27 & 0.47 & 1.28 & 0.25 & 1.45 & 0.23 & 33.92 & 0.44 & 407.75 \\
\hline 25 & & & & & & & & & & & & & 24.85 & 51.57 & 6.67 & 23.73 & 4.90 & 1.22 & 4.42 & 0.80 & 5.00 & 1.08 & 2.99 & 0.49 & 2.96 & 0.43 & 17.01 & 0.78 & 200.56 \\
\hline 26 & 65.07 & 0.35 & 16.19 & 4.42 & 0.07 & 0.07 & 1.86 & 4.16 & 4.10 & 1.31 & 0.17 & 2.02 & 19.64 & 42.59 & 5.33 & 18.32 & 3.05 & 0.92 & 2.02 & 0.29 & 1.30 & 0.26 & 0.69 & 0.13 & 0.72 & 0.11 & 21.08 & 0.70 & 215.56 \\
\hline 27 & 64.97 & 0.43 & 15.87 & 2.75 & 1.46 & 0.47 & 1.29 & 3.86 & 3.41 & 2.64 & 0.25 & 2.10 & 13.16 & 28.22 & 4.06 & 14.35 & 2.74 & 0.87 & 1.92 & 0.30 & 1.26 & 0.25 & 0.64 & 0.12 & 0.57 & 0.09 & 4.86 & 0.79 & 131.11 \\
\hline 28 & 62.23 & 0.43 & 17.54 & 3.16 & 1.90 & 0.55 & 1.58 & 3.85 & 3.07 & 3.05 & 0.25 & 1.66 & 12.14 & 26.10 & 3.63 & 13.14 & 2.36 & 0.81 & 1.59 & 0.23 & 1.01 & 0.19 & 0.47 & 0.08 & 0.37 & 0.05 & & 1.08 & 95.37 \\
\hline 29 & & & & & & & & & & & & & 16.88 & 30.98 & 3.92 & 12.59 & 2.46 & 0.76 & 1.98 & 0.37 & 1.45 & 0.29 & 0.74 & 0.13 & 0.68 & 0.10 & & & \\
\hline 30 & & & & & & & & & & & & & 24.10 & 48.60 & 4.83 & 19.14 & 2.87 & 0.98 & 2.36 & 0.09 & 1.56 & 0.17 & 0.82 & 0.16 & 0.73 & 0.10 & 13.38 & 1.11 & 68.55 \\
\hline 31 & 71.66 & 0.25 & 14.48 & 0.70 & 2.34 & 0.10 & 0.96 & 1.00 & 6.04 & 1.50 & 0.10 & 0.44 & 21.88 & 48.34 & 5.00 & 21.85 & 4.07 & 1.04 & 3.26 & 0.28 & 2.73 & 0.42 & 1.60 & 0.31 & 1.58 & 0.23 & 19.01 & 1.22 & 62.17 \\
\hline
\end{tabular}

keynote: 1: biotite-plagioclase gneiss;

2: plagioclase fels;

3: amphibole gneiss;

4: dolomite-bearing biotite gneiss

5: graphite diopsidite:

6: graphite-diopside marble:
7: graphite-calcite diopsidite;

8: amphibole-albite fels:

9: epidotite:

10: sillimanite-garnet-quartz-microcline gneiss;

11: diopside marble;
12: magnetite-quartz-diopsidite;

13: magnetite-quartz-diopsidite;

14: graphite-calcite-actinolite gneiss:

15: calcite-biotite gneiss:

16: plagioclase quartzite;
17: migmatized quartzite;

18: biotite schist;

9-22: quartz-sericite schis:

23-24: sericite schist;

25: quartz-sericite schist;
26: quartz-biotite schist: 27-28: amphibole-biotite gneiss;

29: mica-quartz schist;

30: amphibole-biotite gneiss:

31: biotite fels. (nomenclature after Winkler, 1976) 


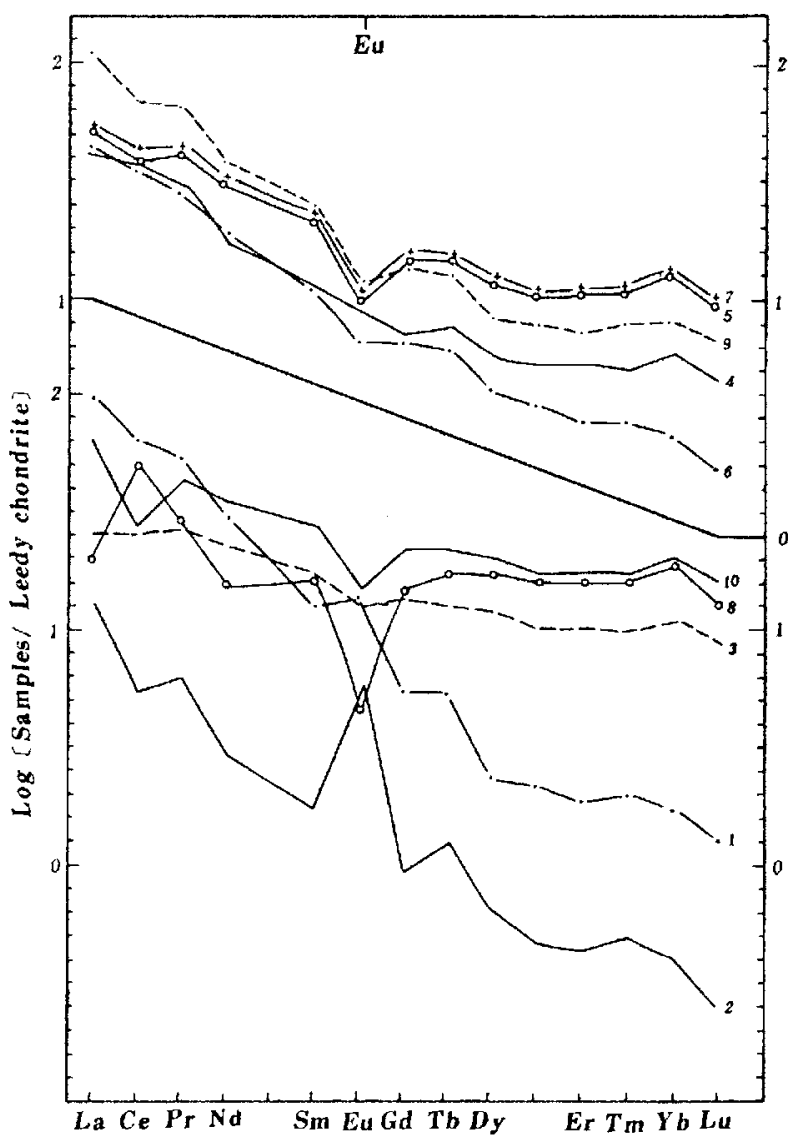

Figure 3 Chondrite-normalized REE patterns for sediments from the Lushan terrain, the upper portion showing the chemical sediments, and the lower showing the clastics.

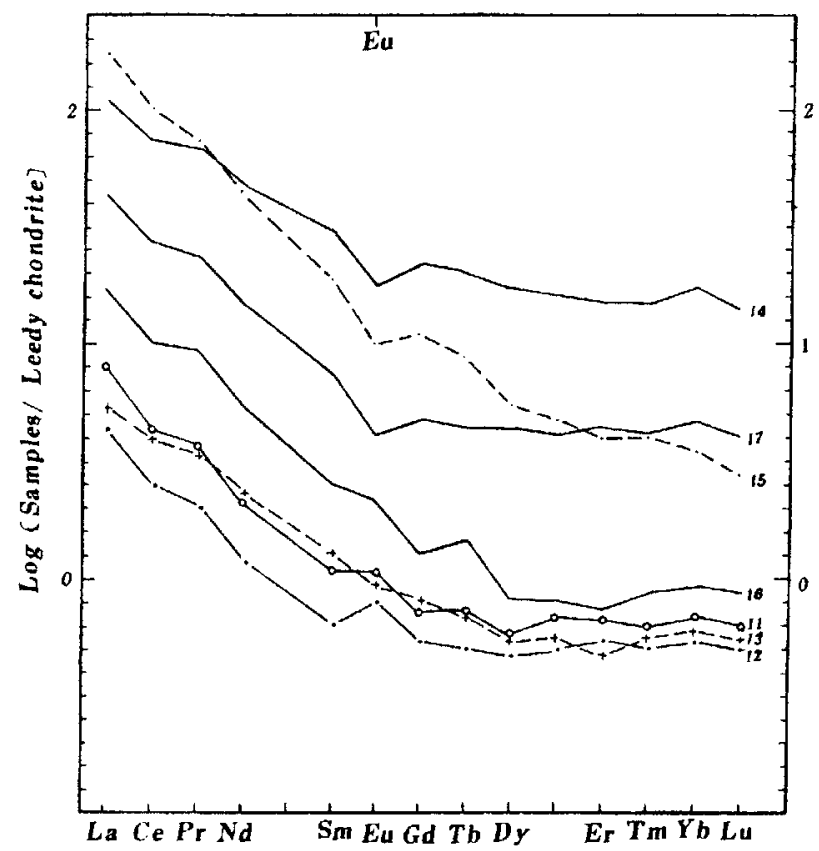

Figure 4 Chondrite-normalized REE patterns for sediments from the Wuyang terrain.

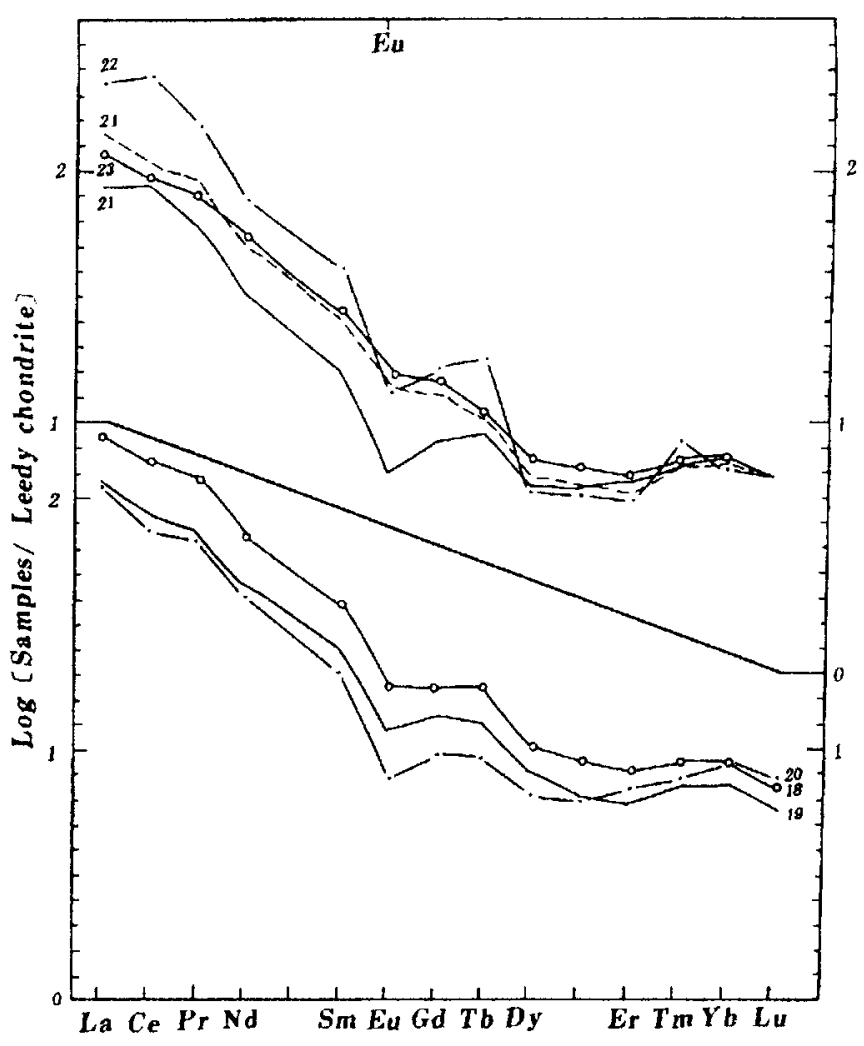

Figure 5 Chondrite-normalized REE patterns for sediments from the Xiaoshan Gp in the Xiaoshan terrain

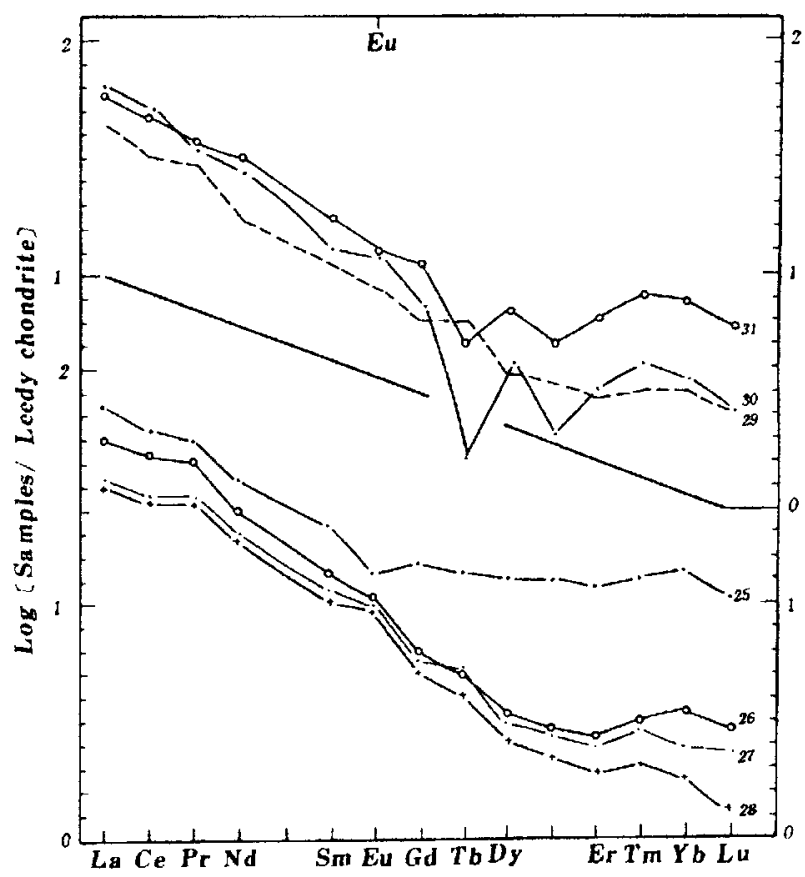

Figure 6 Chondrite-normalized REE patterns for sediments from the Songshan, Jishan and Xiong'ershan terrains. 


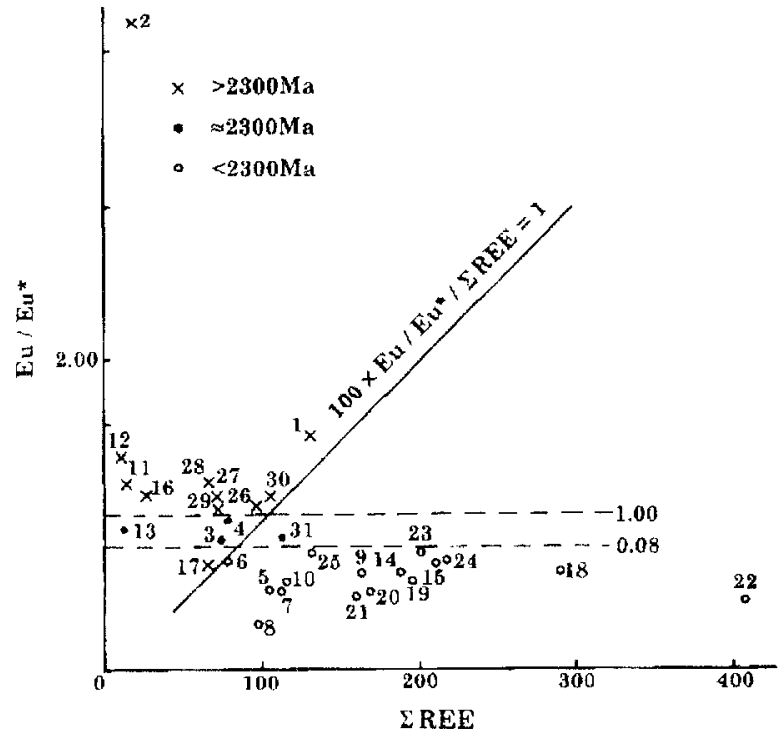

Figure $7 \mathrm{Eu} / \mathrm{Eu}$ * versus $\Sigma R E E$ diagram for the sediments from the southern margin of the North China craton, showing contrasting sedimentary characteristics between pre-2300 Ma and post-2300) Ma.

were collected from the sediments with an age of $2300 \mathrm{Ma}$ and all demonstrated positive Eu-anomaties and low $\sum$ REE (Figure 6).

\section{Jishan terrain}

No.29 is the only sample of the sediment from the Jishan terrain, which was formed before 3,000 Ma (Figure $2 \mathrm{E}$, Table 2). Its REE pattern is quite similar to that of nos. $27-28$ and also marked with low $\sum$ REE and moderately positive Eu-anomaly (Figure 6).

\section{Xiong'ershan terrain}

No. 30, the sediment older than 2,550 Ma in age (Figure 2F, Table 2), displays positive Eu-anomaly (Figure 6). No. 31, the clastic sediment formed at about $2300 \mathrm{Ma}$, shows slight Eu-depletion (Figure 6).

\section{Summary}

Now we can say that the sediments younger than $2300 \mathrm{Ma}$ are consistently depleted in Eu, the sediments older than $2300 \mathrm{Ma}$ are marked by positive Eu-anomalies, and the sediments deposited at about 2300 Ma show slight Eu-depletion or no Eu-anomaly. This conclusion can be directly made from the illustration in Figure 7. The sediments older than $2300 \mathrm{Ma}$ are entirely above the line of $\mathrm{Eu} / \mathrm{Eu}^{*}=1.00$ except No. 17 which was migmatized; the sediments younger than $2300 \mathrm{Ma}$ are all below the line of $\mathrm{Eu} / \mathrm{Eu}^{*}=0.80$; and the sediments formed at about $2300 \mathrm{Ma}$ are plotted between these two lines. In Figure 7. the sediments less than $2300 \mathrm{Ma}$ are distributed on the right side of $100\left(\mathrm{Eu} / \mathrm{Eu}^{*}\right) / \sum \mathrm{REE}=1$; while the sediments more than $2300 \mathrm{Ma}$ lie on the left side of the line. Therfore, the sediments younger than $2300 \mathrm{Ma}$ possess higher $\sum$ REE than those older than $2300 \mathrm{Ma}$ (Table 4).

Table 4 Contrasting in REE geochemical features between sediments earlier than $2300 \mathrm{Ma}$ and those later than $2300 \mathrm{Ma}$

\begin{tabular}{lcccc}
\hline $\begin{array}{l}\text { Age of } \\
\text { sediments }\end{array}$ & $\begin{array}{l}\text { Sample } \\
\text { number }\end{array}$ & Eu/Eu* & EREF & LaN/YbN \\
\hline$>2300 \mathrm{Ma}$ & $9 \#$ & 1.51 & 51.14 & 15.74 \\
$>2300 \mathrm{Ma}$ & 11 & 1.45 & 59.71 & 19.41 \\
$2300 \mathrm{Ma}$ & 4 & 0.90 & 68.38 & 6.61 \\
$<2300 \mathrm{Ma}$ & 16 & 0.60 & 176.97 & 14.83 \\
\hline
\end{tabular}

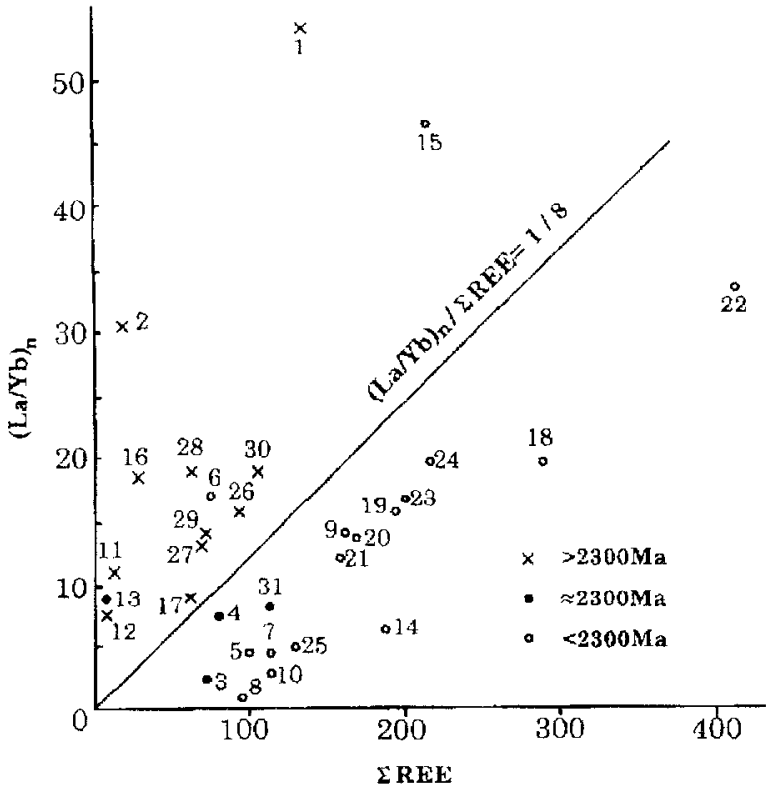

Figure $8 L a_{N} / Y b_{N}$ versus $\sum R E E$ diagram for the sediments from the southern margin of the North China craton, showing contrasting sedimentary characteristics between pre -2,300 $\mathrm{Ma}$ and post-2,300 Ma sediments

\section{Discussion}

The differences in REE patterns between the Archean and the Proterozoic fine-grained clastic sediments (shales) were discussed in details by Taylor and Mclennan (1985) who advanced that development of the K-granites at the end of the Archean had resulted in the Eu-depletion of the post-Archean sediments. According to their model, the REE characteristics of the clastics depend on their provenance. Therefore, the differences in REE in clastic sediments from the pre-2300 Ma and the post-2300 Ma strata should reflect a great tectonic change, i.e., the Archean-Proterozoic transition, occurring at about $2300 \mathrm{Ma}$ in the area in question.

However, at the southern margin of the North China craton, none of $\mathrm{K}$-granite masses older than $2200 \mathrm{Ma}$ is known and the sedimentary Eu-depletion started from about 2300 Ma. Hence the sedimentary Eu-depletion, especially the chemical sedimentary Eudepletion, can not be simply attributed to the formation of $\mathrm{K}$-granite.

The sedimentary Eu-anomaly should be controlled by the nature of Eu and the sedimentary environment. It is well known that $\mathrm{Eu}^{2+}$ and $\mathrm{Eu}^{3+}$ are two natural states of Eu. The value of $\mathrm{Eu}^{3+} / \mathrm{Eu}^{2+}$ must be affected by $f \mathrm{O}_{2}$. Therefore, the sedimentary Eu-anomaly can be illustrated by considering the changes in $f \mathrm{O}_{2}$ (Table 3). When $\mathrm{fO}_{2}$ is low, the aqueous anions will be prevailed by soft bases such as $\mathrm{HS}^{-}, \mathrm{S}^{2-}, \mathrm{SCN}^{-}, \mathrm{S}_{2} \mathrm{O}_{3}{ }^{2-}, \mathrm{CO}, \mathrm{CH}_{4}$; and the value of $\mathrm{Eu}^{3+} / \mathrm{Eu}^{2+}$ is low; Eu is dominated by Eu ${ }^{2+}$. As a kind of acid, $\mathrm{Eu}^{2+}$ is softer than $\mathrm{R}^{3+}$ (trivalent REE ions, including $\mathrm{Eu}^{3+}$ ) which are all typical hard acids. Eu ${ }^{2+}$ is easier than $\mathrm{R}^{3+}$ to combine with soft bases into stable complexes and to precipitate from water. $\mathrm{R}^{3+}$ will prefer to stay in water as ions. Thereby, sediments deposited in the reducing environment should be characterized by low $\sum$ REE and positive Eu-anomaly. When $f \mathrm{O}_{2}$ is high, Eu is dominated by Eu ${ }^{3+}$ and the anions will be hard bases such as $\mathrm{OH}^{-}, \mathrm{SO}_{4}^{2-}, \mathrm{CO}_{3}{ }^{2-}$ and $\mathrm{NO}_{3}{ }^{2+}$. As hard acids, $\mathrm{Eu}^{3+}$ and other $\mathrm{R}^{3+}$ tend to be combined with the hard bases (particularly $\mathrm{OH}^{-}$) into stable complexes and to precipitate; while Eu+ ${ }^{2+}$ is likely to stay in water. Consequently, the sediments deposited under oxidizing conditions should be characterized by high $\Sigma$ REE and Eudepletion.

According to the above-discussed oxidation-reduction mechanism, the sediments, formed after $2300 \mathrm{Ma}$ at the southern margin of 
the North China craton and characterized by high $\Sigma$ REE and negative Eu-anomaly, should have been deposited in the oxidizing environment: whereas those older than 2300 Ma featured by low $\sum$ REE and positive Eu-inomaly should have been deposited in reducing conditions. Hence the oxic atmosphere should have appeared at about $2300 \mathrm{Ma}$.

Becaluse the $\mathrm{LR}^{3+}$ are softer than $\mathrm{HR}^{3+}$, the sediments formed in oxidizing environment should have lower value of $\mathrm{LaN}_{\mathrm{N}} / \mathrm{Yb}_{\mathrm{N}}$ than those in anoxic conditions in the light of the mechanism discussed above and shown in Table 3. In the studied area. the sediments older than $2300 \mathrm{Ma}$ formed in reducing condition, should have higher values of $\mathrm{LaN}_{\mathrm{N}} / \mathrm{Yb}_{\mathrm{N}}$, whereas those later than $2300 \mathrm{Ma}$ deposited in oxidizing environment have lower values of $\mathrm{LaN} / \mathrm{Yb}$, which is in general agreement with the facts shown in Table 4 and Figure 8 . In Figure 8 , the sediments more than and less than 2300 Ma are plotted on the left and the right sides of the line of $(\mathrm{La} / \mathrm{Yb})_{\mathrm{N}} / 2 \mathrm{REE}=1 / 8$. Therefore, again the values of LaN/Y $\mathrm{b}_{\mathrm{N}}$ of the sediments from the southern margin of the North China craton indicate a great change in surficial environment at about $2300 \mathrm{Ma}$

It should be also pointed out that the conclusion on the environmental change at about 2300 Ma can also be supported by the appearance of sulfates. BIFs with high $\mathrm{Fe}^{3+} / \mathrm{Fe}^{2+}$, red beds, glacials and other important changes at about 2300 Ma (Chen, 1989. 1990; Chen at al. 1991b. 1994. 1996: Condie, 1981, 1982; Schidlowski, 1976; Frakes. 1979; Hu. 1988; Eriksson et al., 1978; Hambrey and Harland. 1981).

\section{Conclusions}

(1) At the southern margin of the North China craton, the sediments more than 2300) Ma show positive Eu anomalies; the sediments less than 2300 Ma are characterized by Eu-depletion: and the sediments al about 2300 Ma demonstrate no obvious Eu-anomaly (Eu/Eu: 0.80 to 3.00$)$.

(2) The values of $\sum R E E$ of the sediments less than 2300 Ma are Inuch higher than those of the sediments more than $2300 \mathrm{Ma}$; the values of $\mathrm{L}_{\mathrm{a} N \mathrm{~N}} / \mathrm{Yb}_{\mathrm{N}}$ of the sediments later than $2300 \mathrm{Ma}$ are lower than those of the sediments more than $2300 \mathrm{Ma}$

(3) The differences in REE characteristics between the sediments more $2300 \mathrm{Ma}$ and those less than 2300 Ma possibly resulted from the changes in the sedimentary environment. The sediments more than $2300 \mathrm{Ma}$ (Archean), with low LREE, positive Eu-anomalies and high LalN $/ Y b_{N}$, should have been deposited in the reducing environment; while those less than 2300 Ma (post-Archean), with high $\Sigma R E E$, low $\mathrm{LaN}_{\mathrm{N}} / \mathrm{Yb}_{\mathrm{N}}$ and negative Eu-inomalies (in general, Eu/Eu* $\mathbf{L}^{*}(0.80)$, deposited in the oxidizing environment.

(4) The sedimentary REE geochemical evolution strongly suggests that the oxidic atmosphere appeared at about $2300 \mathrm{Ma}$, which is consistent with that of the studied sulfates, BIFs, red beds, glacials, etc.

(5) Besides the provenance of sediments, the sedimentary environment is the chief factor affecting the REE characteristics of sediments, particularly, of chemical sediments. The sediments formed in the reducing environment should be marked by positive Eu-anomalies, high $\mathrm{LaN}_{\mathrm{N}} / \mathrm{Yb}_{\mathrm{N}}$ and low $\Sigma \mathrm{REE}$; whereas those formed in the oxidiring condition should be characterized by clear Eu-depletion, low $L_{\text {LaN }} / Y_{N}$ and high $\sum R E E$.

\section{Acknowledgements}

The authors thank professors Ouyang Ziyuan, Hu Shouxi, Zhu Jinchu, Qiu Yuzhuo, Fu Shigu and Gu Lianxing for their helpful discussion and reviewing of the manuscript.

\section{References}

Chen, Yanjing. 1987. The Formation and Ivolution of the Crystalline Basement at the Southern Margin of the North China Craton. Thesis for MS degree. Department of Earth Sciences of Nanjing University, 267pp. IIn Chinese with English abstract)

Chen, Yanjing, 1989. Tentative study on Catastrophe in geotogical environment at $2300 \mathrm{Mat}$. in: Progress in Comprehensive Study on the Relationship anong Lile, Earth and Cosmos, Chinese Science \& Technology Press. Beijing, p.78-82. (in Chinese).

Chen. Yanjing, 1990. Evidences for the catastrophe in geofogic enviromment at about $2300 \mathrm{Ma}$ and the discussions on several problems. Journal of Stratigraphy, 14(3):178-186. (in Chinese with English abstract).

Chen. Yanjing, and Fu. Shigu, 1992. Gold Mineralization in West Henan. China. Chinese Seismological Press. 234pp. (in Chinese with English abstract)

Chen. Yanjing, Fu. Shigu, and Hu, Shouxi, 1988. The main element character and its significance of different type greenstone belts at the southern margin of Northern-China craton. J. Nanjing University (Earth Sciences). 1:70-83 (in Chinese with Engtish abstract)

Chen, Yanjing, Fu, Shigu, Hu. Shouxi. Chen, Zeming, and Zhou, Shouzhi, 1991a. The inter-block differences and their metillogenic significance of the southern margin of the North China craton. Geotectonica el Metallogenica, 15(3): 265-271 (with English ahstract).

Chen, Yanjing.. Fu. Shigu, Hu, Shouxi., Chen, Zeming. Zhou, Shunzhi, I.in. Qianlong, and Fu, Guanghong, 1989. Shipaihe movement and the disseetion of "Dengteng Group". I. Statigraphy. 13,81-87. (in Chinese with English abstract).

Chen, Yanjing. Ji, Haizhang. Thou, Xiaoping, and Fu, Shigu, 199/b. The challenge to the traditional geological theories from revelation of the catastrophe at 2300Ma: new knowledge on several important geological subjects. Advance in Earth Science, 6(2): 63-68. (with English abstract)

Chen, Yanjing, Ouyang, Ziyuan. Yang, Qiujian, and Deng, Jian, 1994. A new understanding of the Archean-Proterozoic boundary. Geologic Review. 40(5):483-488. (in Chinese with English abstract)

Chen Yanjing, Zhao Taiping, Yang Zhongfang, Zhang Fasheng, Qu Lili, and Chang 7hioshan, 1996. Current Progresses in Indications of Crustal Composition and Sedimentary Fnviromment and Their Evolutions with Sedimentary Trace Ekements. Geology-(jeochenistry, (3): 1-125. (In Chinese).

Condie. K. C.. 1981. Archean Greenstone Belts. Elsevier, Amsterdan, $4.34 \mathrm{pp}$.

Condie. K. C., 1982. Plate Tectonics \& Crustal Evolution. Pergamon. New York, $310 \mathrm{pp}$

Condie, K.C. 1993. Chemical composition and evolution of the upper continental crust: contrasting results from surface samples and thales. Chemical Geology, 104:1-37.

Dai, Anbang, 1987. Coordination Chemistry. Academic Press. Beijing, 750 $\mathrm{pp}$ (in Chinese)

Eriksson, K. A.. and Truswell, J. F., 1978. Geological process and atmospheric evolution in the Precimbrian. in: D. H. C. Tarling (Editor). Fivolution of the Earth's Crust. Academic Press, London, pp. 219-238.

Frakes, L. A., 1979. Climates Throughout Geologic Time. Elsevier, Amsterdam. $310 \mathrm{pp}$

Hambrey. M.J., and Harland, W.B.(eds), 1981. Earth's PrePleistocene Glacial Record. Cambridge University Prens. London. 1004p.

Hu, Shouxi, 1988. Geology and Metallogeny of the Collision Belts Between the Southern and the Northern China Plate. Nanjing University Press, Nanjing, 558pp. (in Chinese)

Kroner, A., 1981. Precambrian plate tectonics, In: A. Kroner (Editor), Precambrian Plate Tectonics. Elsevier, Amsterdam, pp. 57-90).

Kroner, A., Compston. W., Zhang, G. W., Guo, A. L., and Todt, W., 1988. Age and tectonic setting of late Archean greenstone-gneiss terrain in Henan province, China, as revealed by single-grain zircon dating. Geology, 16: 211-215.

Li, S. G, Hart, S. R., Guo, A. L., and Zhang, G. W.. 1987. A whole-rock SmNd age for the Dengfeng Group in central Henan province and its tectonic implications. Science Report. No.22: 1728-1731. (in Chinese).

Mclennan,S.M., and Taylor.S.R., 1991. Sedimentary rocks and crustal cvolution: tectonic setting and secular trends. The J. of Geol.. 99:1-21.

Schidlowski, M., 1976. Archean atmosphere and evolution of the terrestrial oxygen. in: B. F. Windley (Editor), The Early History of the Earth. Wiley \& Sons, London, pp. 525-535.

Schopf, J. W., 1977. Evidence of Archean life. in: C. Ponnamperuma (edi(or), Chemical Fvolution of the carly Precambrian. Academic Press, New York. pp. 101-105. 
Sun, Dazhong (editor), 1984. The Early Precambrian Geology of Eastern Hebei. Tianjin Science and Technology Press, Tianjin, 273pp. (in Chinese, with English abstract).

Taylor, S. R., and Mclennan, S. M., 1985. The continental crust: Its Composition and Evolution. Blackwell, Oxford, 312pp.

Chen Yanjing, born in 1962, is Associate Professor of Ore Deposits at the Department of Geology, Peking University. Since receiving his $P h . D$ from Nanjing University in 1990, he has been undertaking study on gold deposits of China, metallogenesis and fluidization in collisional orogenesis, and Precambrian geology and mineralization.

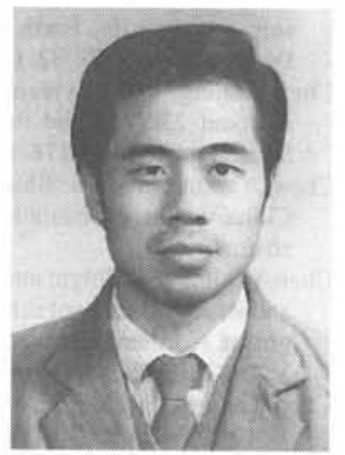

Winkler, H. G. F., 1976. Petrogenesis of Metamorphic Rocks (fourth edition). Springer-Verlag, New York, 334pp.

Zhao Yongchao, born in 1971, was graduated from the Geology Department of Peking University in 1994. Now is a postgraduate student for doctor's degree of mineral deposits in Peking University, mainly on geothermal deposits.

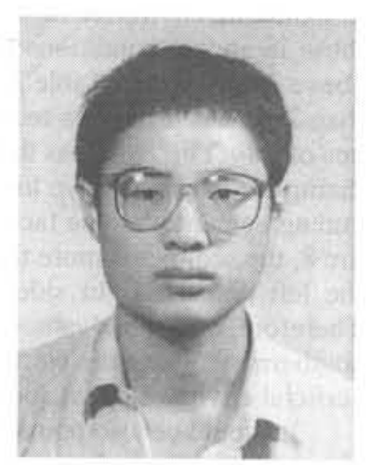

\title{
31st International Geological Congress
}

\author{
August 6-17, 2000 \\ Rio de Janeiro, Brazil
}

The 31st International Geological Congress will be held in Rio de Janeiro, Brazil, August 6-17, in the year 2000. A Preparatory Commission has been working since 1995 to establish the Organizing Committee that will plan the scientific program and related activities of the Congress.

The theme of the Congress is "Geology and Sustainable Development: Challenges for the Third Millenium". Although the principal venue of the Congress is Brazil, other South American countries will be strongly involved in organizing the program and field trips and will host some pre- and post-conference events.

The scientific program will feature comprehensive, multi-disciplinary discussions of the important problems facing the geological sciences, and will strive to organize scientific sessions that reflect the interdisciplinary nature of today's science, emphasizing the importance of geological sciences to human society. In addition to highlighting the theme of the Congress, sessions will focus on frontier subjects and hot spots of international geoscientific research, with special attention to the unique geological conditions of the continent of South America.

Special symposia will feature invited papers dealing with the origin and history of the Earth; geosciences and human survival; environment and natural hazards; global changes and future environment; structure of the lithosphere and deep crustal processes; contemporary lithospheric motion; global tectonic belts; orogenic belts; basin analysis; energy and mineral resources for the 21 st century; new technologies for the geosciences; and progress of international geoscience projects.

The Congress will also include an attractive program of short courses, workshops, and field trips. Field excursions will visit most of the geologic provinces of South America (including Antarctica), and will focus on the characteristic geologic features of the South American continent: major orogenic belts, large-scale sedimentary basins, mineral deposits, and a great variety of other geologic attractions.

The First Circular will be mailed in October, 1997. Further information about the Congress will be posted as it becomes available.

For the present, the principal point of contact is:

Prof. Hernani Chaves

President, Preparatory Commission for the 31 st IGC

Ave. Pasteur, 404

Urca: Cep 22290-204

Rio de Janeiro, BRAZIL

Tel: +55212955337

Fax: +55 21 542-3647

E-mail: Hernani@uerj.br 